\section{JTI}

JOURNAL OF

TRAUMA AND INJURY

\title{
Complete Transection of the Cystic Duct and Artery after Blunt Trauma: A Case Report
}

\author{
Sung Hoon Cho, M.D., Kyoung Hoon Lim, M.D., Ph.D. \\ Trauma Center, Department of Surgery, Kyungpook National University Hospital, School \\ of Medicine, Kyungpook National University, Daegu, Korea
}

Received: November 20, 2021

Revised: December 1, 2021

Accepted: December 7, 2021

\section{Correspondence to}

Kyoung Hoon Lim, M.D., Ph.D. Department of Surgery, Kyungpook National University Hospital, School of

Medicine, Kyungpook National University, 130 Dongdeok-ro, Jung-gu, Daegu 41944, Korea

Tel: $+82-53-420-5605$

Fax: +82-53-421-0510

E-mail:drlimkh@knu.ac.kr

ORCID: https://orcid.org/0000-00026842-7129
Extrahepatic biliary tract and gallbladder injuries following blunt abdominal trauma are uncommon. Traumatic cystic duct transection is even rarer, which has frequently caused missed diagnosis and delayed treatment. An 18-year-old female patient with no past medical history was transferred to the Trauma Center of Kyungpook National University Hospital after falling from a height of approximately 20 meters. She became hemodynamically stable after initial resuscitation, and initial contrast-enhanced abdominal computed tomography (CT) showed right kidney traumatic infarction and multiple intrahepatic contusions with minimal fluid collection but no extravasation of the contrast. She was admitted to the intensive care unit. On the second day of hospitalization, her abdomen became distended, with follow-up CT showing a large collection of intra-abdominal fluid. Laparoscopic exploration was then performed, which revealed devascularization of the gallbladder with complete transection of the cystic duct and artery. Laparoscopic cholecystectomy was performed, as well as primary closure of the cystic duct orifice on the common bile duct using a 4-0 Prolene suture. After surgery, no clinical evidence of biliary leakage or common bile duct stricture was observed.

Keywords: Cystic duct; Gallbladder; Blunt trauma

\section{INTRODUCTION}

Traumatic injuries of the extrahepatic bile duct, which are mainly caused by penetrating abdominal trauma, are quite infrequent [1]. Similarly, gallbladder injuries caused by blunt abdominal trauma rarely occur [2]. Gallbladder injuries mainly involve perforation of the body or avulsion from its hepatic bed. There are only few reports on avulsion of the cystic duct and artery [2]. This uncommon injury can be difficult 
to diagnose, and it is often the case that no diagnosis is established preoperatively. We herein report a case of a cystic duct and artery transection after a fall and review the relevant literature.

\section{CASE REPORT}

An 18-year-old female patient with no past medical history was transferred to the Trauma Center of Kyungpook National University Hospital after falling from a height of 20 meters. Upon arriving at the emergency room, she was hemodynamically stable after initial resuscitation. She complained of mild abdominal pain and had mild tenderness on right upper quadrant abdominal area. Contrast-enhanced abdominal computed tomography (CT) showed partial infarction of the right kidney (grade III), multiple intrahepatic contusion without contrast extravasation (grade III) and a small amount of fluid collection in the perihepatic space and the pouch of Douglas (Fig. 1). Other trauma series showed fractures of the right humerus and left radius, with an Injury Severity Score of 14 . Hence, conservative treatment in the traumatic intensive care unit was considered.

On the second day after admission, her abdomen became distended, but her symptoms and other physical examination findings remained the same. In addition, the vital signs were stable and there was no change in the hemoglobin level; however, the creatinine level had increased. Follow-up abdominal CT without contrast enhancement was performed, which showed a large collection of intra-abdominal fluid greater than that observed on initial CT, but no free air (Fig. 2). Since the patient complained of discomfort due to abdominal distension, paracentesis was performed to alleviate discomfort and diagnosis. After abdominal paracentesis, approximate-

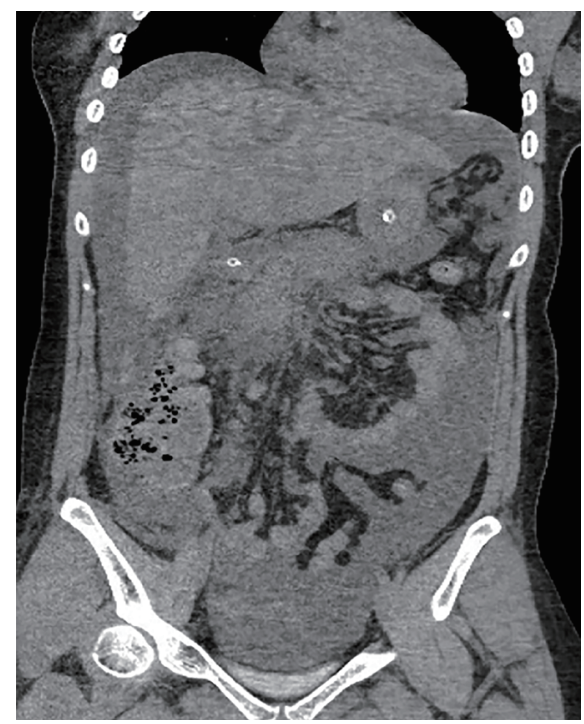

Fig. 2. Follow-up abdominal computed tomography without contrast enhancement showing a large collection of intra-abdominal fluid.
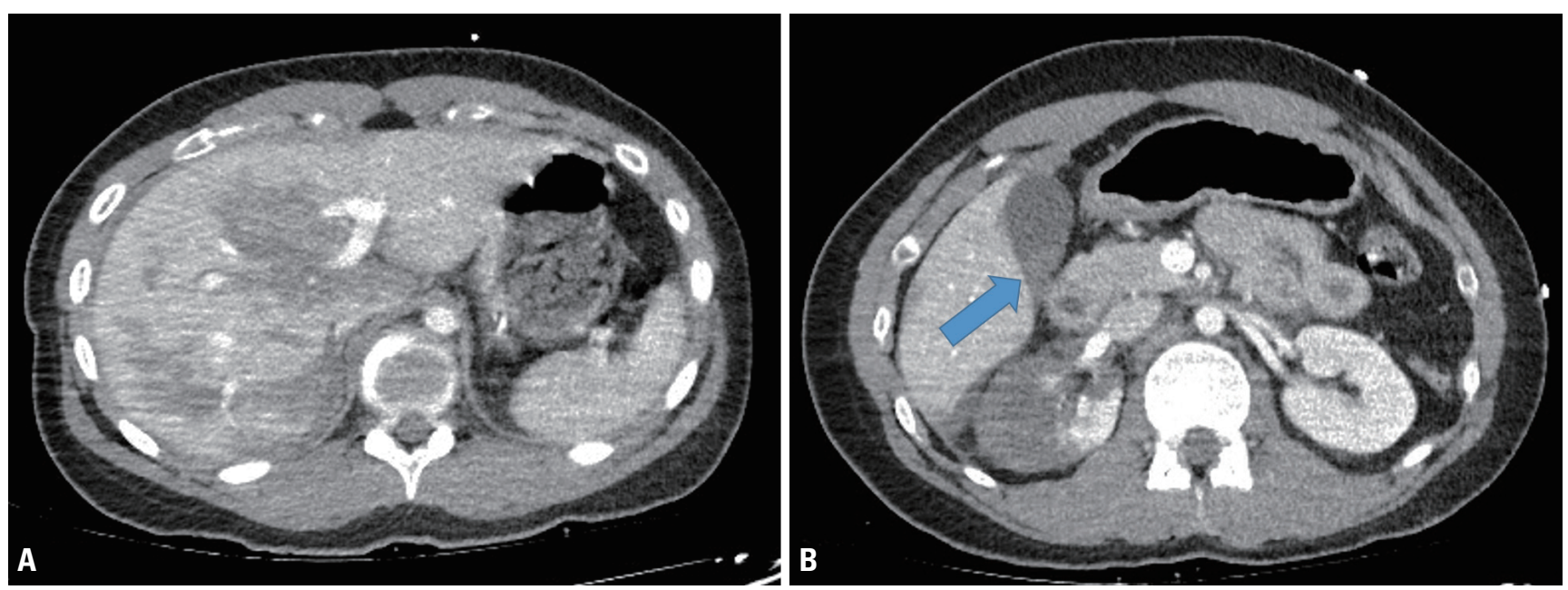

Fig. 1. Initial contrast-enhanced abdominal computed tomography. (A) Multiple intrahepatic contusion without contrast extravasation. (B) Poor enhancement of the gallbladder wall and high density lesion thought to be hematoma in the gallbladder (arrow). Partial infarction of the right kidney and a small amount of fluid collection in the perihepatic space. 
ly 2,000 $\mathrm{mL}$ of bilious and slightly bloody ascites was drained. Based on the aforementioned information, perforation of the upper jejunum was suspected. For this reason, laparoscopic exploration was subsequently performed, which revealed necrosis of the gallbladder without bowel injury. The infundibular portion of the gallbladder was detached from the hepatic bed, and the cystic duct and artery were already transected completely. The cystic duct was avulsed at the orifice of the common bile duct (CBD), causing bile to spill through the avulsion site. Laparoscopic cholecystectomy and primary closure of the avulsion site on the CBD were performed using a 4-0 Prolene suture (Fig. 3). After her surgery, no complications, including biliary leakage or CBD stricture, were observed, and the patient was discharged on the 38th day of hospitalization after orthopedic surgery.

\section{DISCUSSION}

Traumatic injuries of the extrahepatic bile duct are quite uncommon, with an incidence rate of $2 \%$ to $5 \%$, and are mostly caused by penetrating abdominal trauma [1]. A collective review of 5,670 cases of blunt and penetrating trauma by Penn showed that traumatic gallbladder injuries had an incidence rate of 1.9\% [3]. Moreover, Gottesman et al. [4] reported that gallbladder injuries accounted for $2 \%$ to $3 \%$ of all blunt abdominal traumas.

The early diagnosis of a bile duct or gallbladder injury is often difficult in a patient with multiple injuries and de-
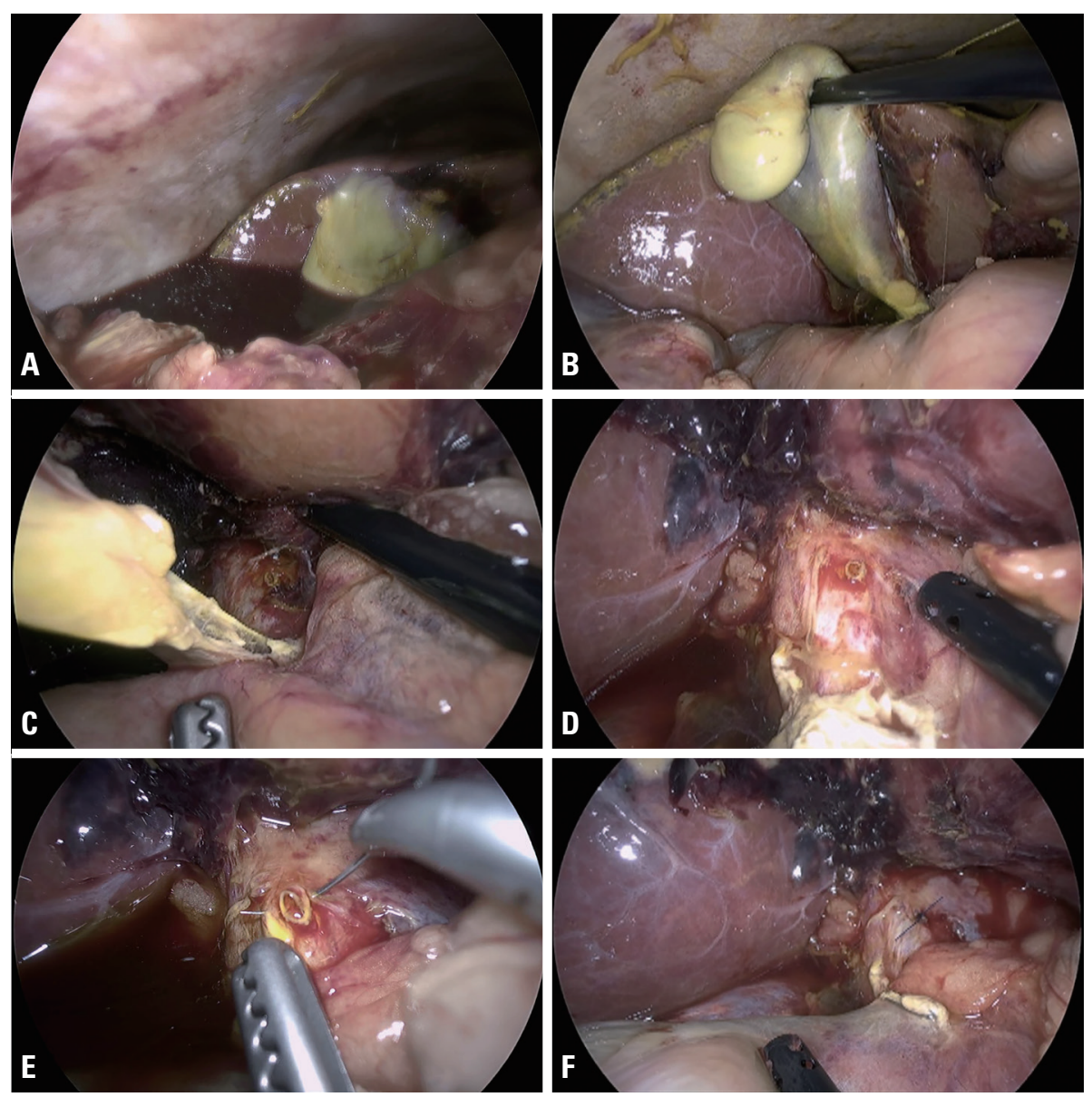

Fig. 3. Laparoscopic exploration. (A) Bilious and bloody ascites. (B) The gallbladder was necrotic and not detached from the hepatic bed. (C) The hepatoduodenal ligament around cystic duct was avulsed and the common bile duct was exposed. (D) The cystic duct was avulsed at the junction of the common bile duct. (E, F) Suture repair of the avulsed opening. 
mands a high index of suspicion. Normal uninfected bile may not cause severe peritonitis; therefore, patients may not manifest clinical symptoms such as fever or severe abdominal pain until weeks later. Atypical symptoms, such as abdominal discomfort, distension, nausea, vomiting, mild ileus, hyperbilirubinemia, and low-grade fever, have been commonly associated with bile duct injury, but are nonspecific. The rarity and atypical clinical manifestation of this entity make an early diagnosis difficult.

The CT findings of gallbladder injury are largely nonspecific. Pericholecystic or perihepatic fluid collection is most common, but is least specific, and may even not present well in imaging performed immediately after injury. Other signs of gallbladder injury are an ill-defined contour of the gallbladder wall, intraluminal high-den-
Table 1. Types of gallbladder injury according to the classification established by Losanoff and Kjossev [6]

\begin{tabular}{|ll|}
\hline Type & \multicolumn{1}{c|}{ Gallbladder injury } \\
\hline IA & Contusion with intramural hematoma \\
\hline B & Contusion with perforation \\
\hline 2 & Rupture \\
3A & Avulsion with partial detachment \\
3B & Avulsion with complete detachment from the liver but with \\
& $\begin{array}{ll}\text { attachment to the structures of the hepatoduodenal } \\
\end{array}$ \\
3C & Tornament \\
3D & Comply from the hepately torn from all attachments \\
4A & Traumatic cholecystitis, secondary to hemobilia \\
4B & Acute acalculus cholecystitis \\
5 & Mucosal tear with leakage of bile \\
\hline
\end{tabular}

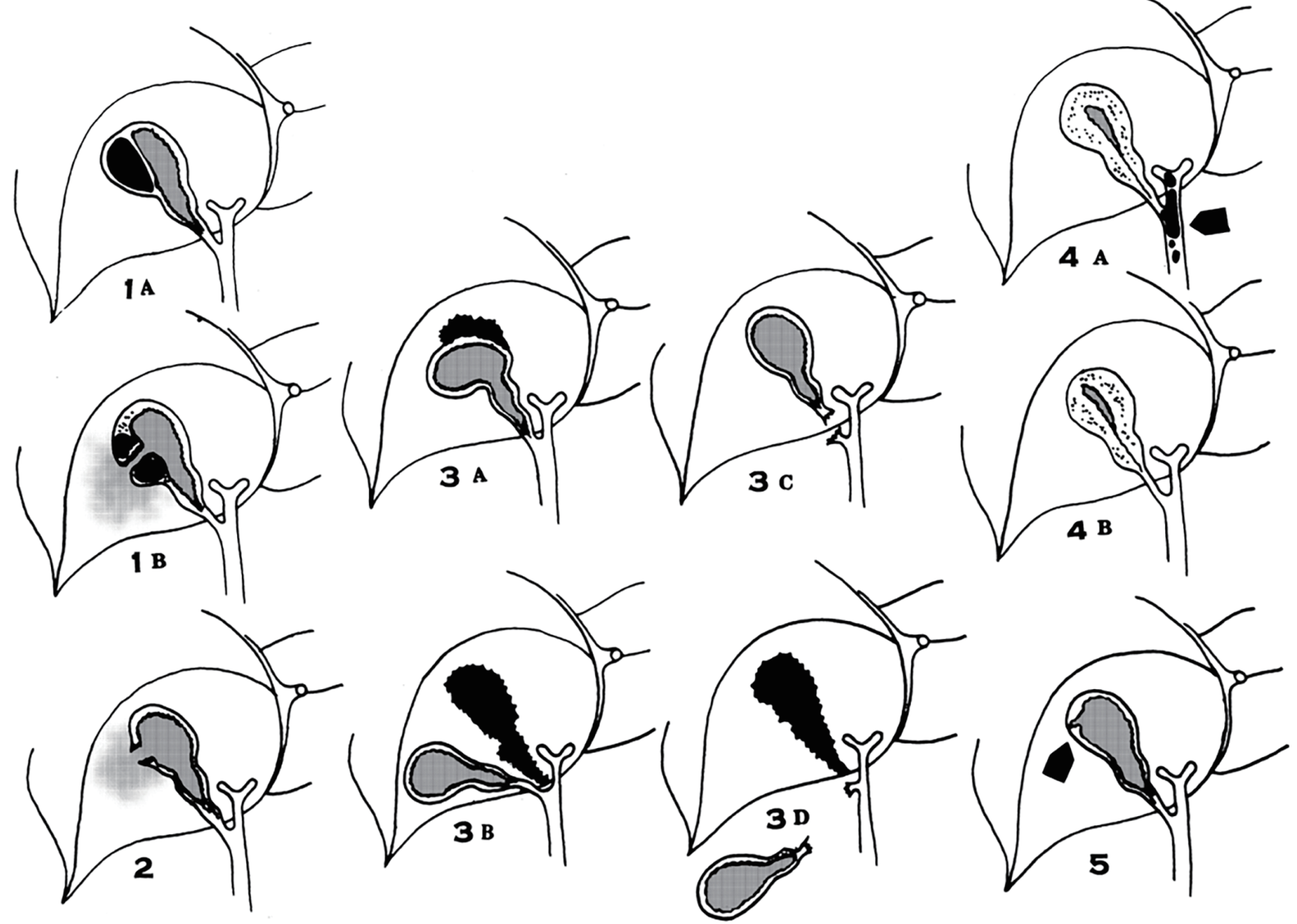

Fig. 4. Types of gallbladder injury according to the classification established by Losanoff and Kjossev [6]. 
sity material (blood), and a thickened gallbladder wall. Moreover, major liver injury often predominates the CT findings and masks subtle abnormalities in the gallbladder [5]. In our case, liver laceration was the main injury, which prevented the early recognition of the gallbladder injury. However, a retrospective review of the initial CT revealed poor enhancement of the gallbladder wall and high-density lesion thought to be hematoma in the gallbladder. Nonetheless, it would have been very difficult to detect cystic artery transection based on this finding alone since enhancement of the gallbladder wall is not clearly visible even in a normally distended gallbladder. Diagnostic peritoneal lavage may help in diagnosing this injury considering that the presence of bile matter may indicate a biliary or upper jejunum injury. In our patient, surgical exploration was performed based on imaging findings suggesting an unexplained increase in intra-abdominal fluid and bilious fluid removed through paracentesis.

Losanoff and Kjossev [6] described a detailed classification of blunt gallbladder injuries (Fig. 4). Accordingly, blunt gallbladder injuries can be classified as contusion, perforation, or avulsion. Perforation, in the form of rupture or laceration, is the most commonly reported blunt gallbladder injury. Avulsion is described as detachment of the gallbladder from the hepatic bed with or without cystic duct and artery injury. According to their classification, our patient had a type $3 \mathrm{C}$ injury (tearing only from the hepatoduodenal ligament) (Table 1 and Fig. 4). Although gallbladder injuries have been reported in several studies, no report has yet described cystic duct and artery transection without avulsion from the hepatic bed [7].

Most perforation sites occur at the dome and neck of the gallbladder. Structurally, these are the weakest points to direct blows [4]. A sudden increase in intraluminal pressure may explain perforations occurring at this location, with the area of greatest diameter having the greatest wall tension [2]. Common causes of blunt trauma resulting in gallbladder injury include motor vehicle accidents, falls, and direct blows [8]. In our case, the infundibulum of the gallbladder was relatively redundant, suggesting that avulsion at the CBD junction was caused by shear force acting between the CBD anchored in the retroperitoneum and the redundant cystic duct and artery.

Given that gallbladder injuries are uncommon and difficult to diagnose early, trauma surgeons need to be cognizant of the possibility of this injury. In patients with unexplained increases in free fluids, a laparoscopic exploration should be considered. Prompt treatment significantly affects the patient's prognosis.

\section{CONFLICTS OF INTEREST}

No potential conflict of interest relevant to this article was reported.

\section{INFORMED CONSENT}

Informed consent was obtained from all individual participants included in this study.

\section{REFERENCES}

1. Ivatury RR, Rohman M, Nallathambi M, Rao PM, Gunduz Y, Stahl WM. The morbidity of injuries of the extra-hepatic biliary system. J Trauma 1985;25:967-73.

2. Salzman S, Lutfi R, Fishman D, Doherty J, Merlotti G. Traumatic rupture of the gallbladder. J Trauma 2006;61:454-6.

3. Penn I. Injuries of the gall-bladder. Br J Surg 1962;49:636-41.

4. Gottesman L, Marks RA, Khoury PT, Moallem AG, Wichern WA Jr. Diagnosis of isolated perforation of the gallbladder following blunt trauma using sonography and CT scan. J Trauma 1984;24:280-1.

5. Pavlidis TE, Lalountas MA, Psarras K, Symeonidis NG, Tsitlakidis A, Pavlidis ET, et al. Isolated complete avulsion of the gallbladder (near traumatic cholecystectomy): a case report and review of the literature. J Med Case Rep 2011;5:392.

6. Losanoff JE, Kjossev KT. Complete traumatic avulsion of the gallbladder. Injury 1999;30:365-8.

7. Khan MR, Begum S. Isolated gallbladder injury from blunt abdominal trauma: a rare co-incidence. J Pak Med Assoc 2020;70(Suppl 1):S95-8.

8. Soderstrom CA, Maekawa K, DuPriest RW Jr, Cowley RA. Gallbladder injuries resulting from blunt abdominal trauma: an experience and review. Ann Surg 1981;193:60-6. 